\title{
Tendon vibration attenuates superficial venous vessel response of the resting limb during static arm exercise
}

\author{
Anna Ooue ${ }^{1 *}$, Kohei Sato ${ }^{1}$, Ai Hirasawa ${ }^{2}$ and Tomoko Sadamoto ${ }^{1}$
}

\begin{abstract}
Background: The superficial vein of the resting limb constricts sympathetically during exercise. Central command is the one of the neural mechanisms that controls the cardiovascular response to exercise. However, it is not clear whether central command contributes to venous vessel response during exercise. Tendon vibration during static elbow flexion causes primary muscle spindle afferents, such that a lower central command is required to achieve a given force without altering muscle force. The purpose of this study was therefore to investigate whether a reduction in central command during static exercise with tendon vibration influences the superficial venous vessel response in the resting limb.

Methods: Eleven subjects performed static elbow flexion at 35\% of maximal voluntary contraction with (EX + VIB) and without (EX) vibration of the biceps brachii tendon. The heart rate, mean arterial pressure, and rating of perceived exertion (RPE) in overall and exercising muscle were measured. The cross-sectional area (CSA vein) and blood velocity of the basilic vein in the resting upper arm were assessed by ultrasound, and blood flow (BF vein) was calculated using both variables.

Results: Muscle tension during exercise was similar between EX and EX + VIB. However, RPEs at EX + VIB were lower than those at EX $(P<0.05)$. Increases in heart rate and mean arterial pressure during exercise at $\mathrm{EX}+\mathrm{VIB}$ were also lower than those at EX $(P<0.05)$. $C S A_{\text {vein }}$ in the resting limb at EX decreased during exercise from baseline $(P<0.05)$, but $C S A_{\text {vein }}$ at $E X+V I B$ did not change during exercise. $C S A_{\text {vein }}$ during exercise at $E X$ was smaller than that at $E X+V I B(P<0.05)$. However, $B_{\text {vein }}$ did not change during the protocol under either condition. The decreases in circulatory response and RPEs during EX $+V I B$, despite identical muscle tension, showed that activation of central command was less during $E X+V I B$ than during EX. Abolishment of the decrease in $C_{\text {vein }}$ during exercise at $E X+V I B$ may thus have been caused by a lower level of central command at $E X+V I B$ rather than EX.
\end{abstract}

Conclusion: Diminished central command induced by tendon vibration may attenuate the superficial venous vessel response of the resting limb during sustained static arm exercise.

Keywords: Central command, Ultrasound technique, Venoconstriction, Venous return

\footnotetext{
* Correspondence: aoue@jwcpe.ac.jp

${ }^{1}$ Research Institute of Physical Fitness, Japan Women's College of Physical

Education, 8-19-1 Kitakarasuyama, Setagaya-ku, Tokyo 157-8565, Japan

Full list of author information is available at the end of the article
} 


\section{Background}

Venomotor response is considered to play an important role in the transfer of blood from veins to the heart. Many studies have suggested that sympathetic activation has an impact on the venomotor responses in the resting limb during static exercise [1-3] and dynamic exercise [1,4-6], since especially superficial venous vessels have rich innervation of sympathetic nerves $[7,8]$ and an operation of sympathectomy [1] and a dosage of $\alpha$-blocking agent clearly abolishes the venoconstriction [2] observed during exercise.

Sustained static exercise produces significant activation of the sympathetic nervous system, and the sympathetic activation during exercise is governed by both central (that is, central command) and peripheral (that is, muscle metaboreflex and mechanoreflex) mechanisms [9-16]. Metabolically sensitive afferents within exercising skeletal muscle detect the buildup of metabolites and act through cardiovascular centers to produce a muscle metaboreflex $[11,13]$. A component of this muscle reflex may arise from muscle mechanoreflex afferents [10]. Duprez and colleagues reported that post-exercise muscle ischemia produces a significant decrease in venous volume in the contralateral limb, and consequently suggested the importance of muscle metaboreflex on venomotor tone in the non-exercising limb during exercise [17].

In addition to muscle metaboreflex, an essential central command role has emerged from a preliminary study [2]. The concept of central command has been conventionally defined as feed-forward control. When a motor command is sent to a muscle, a parallel or collateral command is sent to cardiovascular centers in the brainstem, and this acts to activate sympathetic nerve activity. Indeed, Lorentsen found an anticipatory increase in the venous pressure in the contralateral limb before the onset of exercise [2], indicating that feedforward control of the central command plays an important role in venous tone. However, the study did not examine the influence of central command during actual exercise [2]. Recent studies have also reported that central command also functions as feedback control, in which somatosensory signals arising from the working muscles continuously provide a feedback signal and probably modulate cardiovascular responses via alterations of perception of effort or effort sense [18-20]. If central command has the function of feedback control, the influence of central command will appear not only before exercise but also during sustained and later periods of exercise. However, this question has not yet been challenged. Verifying the influence of central command on the venous tone during actual and sustained static exercise is therefore necessary. On the other hand, using the non-invasive ultrasound Doppler method [21-24], assessment of a single vein response during exercise can extend prior knowledge about the influence of central command on the venous system during exercise.

Based on these considerations, we investigated whether central command affects venomotor tone in the contralateral limb during sustained static exercise. In the present study, using vibration of the biceps brachii tendon reported previously $[14,25]$, we evaluated whether less activation of central command during sustained static elbow flexion accompanies lower responses of the cross-sectional area of the superficial vein in the resting upper arm $\left(\mathrm{CSA}_{\text {vein }}\right)$. Tendon vibration during active muscle contraction excites the primary afferents of muscle spindles of the contracting muscle, thereby inducing reflex tension via the monosynaptic tendon reflex, which in turn aids voluntary tension development and consequently reduces the amount of central command required to generate a given force $[14,25,26]$. In addition, tendon vibration was also useful for investigating the influence of central command without inducing discomfort or nociceptor afferent input [14].

\section{Methods \\ Subjects}

Eleven healthy subjects (three males and eight females) volunteered to participate in the study. Their mean \pm standard deviation age, height, and weight were $21.3 \pm$ 0.9 years, $165.0 \pm 6.6 \mathrm{~cm}$, and $55.2 \pm 5.7 \mathrm{~kg}$, respectively. All subjects were nonsmokers. The participants were asked not to drink beverages containing caffeine or alcohol for 24 hours and not to eat for at least 2 hours before the start of the experiment. The purpose, procedures, and risks of the study were explained to the subjects, and their informed consent was obtained. The study was approved by the Human Ethics Committee of the Japan Women's College of Physical Education and was conducted in accordance with the Declaration of Helsinki.

\section{Muscle tendon vibration and maximal voluntary contraction}

Before the main protocol, the subjects were examined to establish the force produced by tendon vibration at rest. A custom vibrator (DPS-380; Dia Medical, Tokyo, Japan) was used to induce left biceps brachii muscle contraction by reflex stimulation of the biceps brachii distal tendon on the cubital fossa [26]. The oscillating frequency of the vibrator was $100 \mathrm{~Hz}$ and its amplitude was $0.8 \mathrm{~mm}$. On the same day, the subjects performed two maximal voluntary static elbow flexions of the left arm using a computer-based multifunctional dynamometer (VINE, Tokyo, Japan) to determine their maximal voluntary contraction (MVC) strength, defined as the highest value obtained in the two trials. 


\section{Experimental protocol}

In a room maintained at $25.1 \pm 0.2^{\circ} \mathrm{C}$, each subject stayed in a semi-reclined position in a chair in which body position could be maintained, while the left elbow was kept at a $90^{\circ}$ angle on a padded armrest with the wrist attached to an arm lever by a Velcro strap. The subjects rested for at least 20 minutes before data collection began. After baseline data were collected for $5 \mathrm{~min}$ utes, subjects performed: static elbow flexion at 35\% MVC without vibration of the biceps tendon for 2 minutes (EX); and static elbow flexion at 35\% MVC with vibration of the biceps tendon for 2 minutes (EX + VIB). Each exercise period was followed by a recovery period of 1 minute. Static elbow flexion was produced using the same dynamometer that was used to measure the MVC (VINE), with visual feedback of the achieved force provided via an oscilloscope display. For EX + VIB, tendon vibration was initiated 1 minute before starting exercise and continued during the exercise. Immediately after exercise, subjects read instructions for the 6 to 20 rating of perceived exertion (Overall RPE) category scale developed by Borg [27] and instructions for rating muscle fatigue sensation (Arm RPE) on a scale of 1 to 10 [28]. In all trials, subjects regulated their respiratory frequency at 10 or 15 breaths/minute using a metronome, because exercise movement and respiratory cycle influence sympathetic nervous system activity. EX and EX + VIB were performed randomly, and the rest period between the two conditions was at least 20 minutes.

\section{Measurements}

Beat-to-beat changes in arterial pressure were assessed by finger photoplethysmography (Finometer; Finapres Medical Systems BV, Arnhem, the Netherlands). The monitoring cuff was placed around the middle finger. The heart rate (HR) and mean arterial pressure (MAP) were determined from the blood pressure waveform using the Modelflow software program, taking into account sex, age, height, and weight (BeatScope 1.1; Finapres Medical Systems BV).

Muscle oxygenation (oxyhemoglobin (oxy- $\mathrm{Hb}$ ) and deoxyhemoglobin (deoxy-Hb) concentration) in the left exercising upper arm and right resting forearm was monitored using a near-infrared spectroscopy system (NIRO-200; Hamamatsu Photonics, Hamamatsu, Japan) at dual wavelengths $(760 \mathrm{~nm}$ and $850 \mathrm{~nm})$. The nearinfrared spectroscopy probe consisted of an optically dense holder containing an emission and detection probe and was secured to the skin with tape to minimize extraneous light.

To measure blood velocity $\left(\mathrm{V}_{\text {vein }}\right)$ and cross-sectional area (CSA), non-invasive ultrasound imaging of the basilic vein (superficial vein) of the resting upper arm was performed 5 to $6 \mathrm{~cm}$ proximal to the cubitus using an
8.7-MHz linear array transducer (Vivid e; GE Healthcare Japan, Tokyo, Japan). A large quantity of ultrasound transmission gel was used to prevent direct contact with the skin and to avoid compression of the vein. $\mathrm{V}_{\text {vein }}$ and CSA were simultaneously measured on a transverse scan of the vein with the transducer tilted at $60^{\circ}$. Positioning of the transducer was determined at the beginning of each experiment, and it remained unchanged to limit potential errors in Doppler angle. $V_{\text {vein }}$ was the result of the mean velocity of spectral Doppler recording every 12 seconds. CSA was calculated by manually tracing the edge of the offline transverse venous image at an arbitrary three points every 12 seconds, and then the three CSA values were averaged. Because CSA was obtained from the image measured at $60^{\circ}$, an accurate CSA $\left(\mathrm{CSA}_{\text {vein }}\right)$ was determined as follows:

$$
\mathrm{CSA}_{\text {vein }}\left(\mathrm{cm}^{2}\right)=\mathrm{CSA} \times \sin 60^{\circ}
$$

$\mathrm{BF}_{\text {vein }}$ in the basilic vein was calculated according to the following formula:

$$
\mathrm{BF}_{\text {vein }}(\mathrm{ml} / \mathrm{min})=\mathrm{V}_{\text {vein }} \times \mathrm{CSA}_{\text {vein }}
$$

\section{Data analysis and statistical analysis}

The HR, MAP, muscle oxygenation, $\mathrm{CSA}_{\text {vein }}, \mathrm{V}_{\text {vein }}$, and $\mathrm{BF}_{\mathrm{vein}}$ were averaged for 61 to 240 seconds before commencing exercise to establish a baseline value. The relative change in these variables from baseline during exercise and the recovery period was calculated. Data are expressed as mean \pm standard error values.

To compare the time-course changes, two-way analysis of variance with repeated measures was applied to the circulatory responses, $\mathrm{CSA}_{\text {vein }}, \mathrm{V}_{\text {vein }}$, and $\mathrm{BF}_{\mathrm{vein}}$ under each condition (EX and EX + VIB), using time and condition as fixed factors. If a main effect of condition and/or interaction was detected, post hoc analysis with a paired $t$ test was performed; and if a main effect of time was detected, post hoc analysis with a Bonferroni test was performed. To compare the baseline data of the circulatory response, $\mathrm{CSA}_{\text {vein }}, \mathrm{V}_{\text {vein }}$, and $\mathrm{BF}_{\text {vein }}$ between EX and EX + VIB, a paired $t$ test was performed. In addition, differences in Overall RPE and Arm RPE between conditions were evaluated by paired $t$ test. $P<0.05$ was considered significant.

\section{Results}

Vibration of the biceps tendon for 2 minutes elicited a reflex force equivalent to $5.3 \pm 2.3 \%$ of MVC. However, the HR (from $63 \pm 3$ beats/minute to $64 \pm 2$ beats/minute), MAP (from $78 \pm 3 \mathrm{mmHg}$ to $78 \pm 3 \mathrm{mmHg}$ ), $\mathrm{CSA}_{\text {vein }}$ (from $0.20 \pm 0.03 \mathrm{~cm}^{2}$ to $0.20 \pm 0.03 \mathrm{~cm}^{2}$ ), $\mathrm{V}_{\text {vein }}$ (from $4.1 \pm 0.5 \mathrm{~cm} /$ second to $3.9 \pm 0.5 \mathrm{~cm} /$ second), and 
$\mathrm{BF}_{\text {vein }}$ of the basilic vein (from $51.6 \pm 10.3 \mathrm{ml} /$ minute to $52.7 \pm 11.9 \mathrm{ml} /$ minute) did not change.

There were no significant differences in the baseline data of circulatory responses, $\mathrm{CSA}_{\text {vein }}, \mathrm{V}_{\text {vein }}$, and $\mathrm{BF}_{\text {vein }}$ between EX and EX + VIB (Table 1).

Muscle tension during EX was similar to that during EX + VIB (Figure 1). However, both Overall RPE and Arm RPE after EX + VIB were significantly lower than after EX (Overall RPE: $11.5 \pm 0.2$ vs. $12.6 \pm 0.3, P<0.05$; Arm RPE: $3.2 \pm 0.3$ vs. $4.9 \pm 0.4, P<0.05)$.

Figure 2 shows the time courses of HR, MAP, and $\mathrm{CSA}_{\text {vein }}, \mathrm{V}_{\text {vein }}$, and $\mathrm{BF}_{\text {vein }}$ of the resting upper arm during $E X$ and EX + VIB. The increase in HR during exercise from 96 to 120 seconds at EX + VIB was less than that at EX (values at 120 seconds of exercise: $39.1 \pm 4.0 \%$ vs. $50.0 \pm 5.9 \%, P<0.05)$ (Figure 2A). Likewise, the increase in MAP during exercise from 96 to 120 seconds at EX + VIB was lower than that at EX (values at 120 seconds of exercise: $26.0 \pm 3.7 \%$ vs. $29.6 \pm 3.2 \%, \quad P<0.05)$ (Figure 2B). $\mathrm{CSA}_{\text {vein }}$ during exercise at EX decreased from baseline (values at 120 seconds of exercise: $-22.9 \pm$ 6.7\%, $P<0.05)$, but $\mathrm{CSA}_{\text {vein }}$ during $\mathrm{EX}+\mathrm{VIB}$ did not change from baseline throughout the protocol. In addition, $\mathrm{CSA}_{\text {vein }}$ at 120 seconds of exercise and during recovery at EX was lower than at EX + VIB $(P<0.05)$ (Figure $2 \mathrm{C}) . \mathrm{V}_{\text {vein }}$ and $\mathrm{BF}_{\text {vein }}$ did not change from baseline, and this response was similar under both conditions (Figure 2D,E).

Figure 3 shows the time courses of muscle oxygenation of the exercising upper arm and resting forearm during EX and EX + VIB. In the exercising upper arm, $\Delta$ oxy-Hb decreased from baseline during exercise and increased from baseline during recovery in both EX and $\mathrm{EX}+\mathrm{VIB}(P<0.05)$. $\Delta$ oxy-Hb of the exercising upper arm during recovery at EX was lower than at EX + VIB $(P<0.05$; Figure $3 \mathrm{~A})$. In the exercising upper arm, $\Delta$ deoxy-Hb increased from baseline during exercise $(P<0.05)$ and returned to baseline during recovery in both EX and EX + VIB (Figure 3B). In the resting forearm, $\Delta$ oxy-Hb and $\Delta$ deoxy-Hb did not change from baseline at both EX and EX + VIB (Figure 3C,D). However, $\triangle$ deoxy- $\mathrm{Hb}$ of the resting forearm during recovery at EX was lower than at EX + VIB $(P<0.05)$.

Table 1 Baseline data under each condition

\begin{tabular}{lcc}
\hline & EX & EX + VIB \\
\hline Heart rate (beats/minute) & $62 \pm 3$ & $64 \pm 3$ \\
Mean arterial pressure (mmHg) & $84 \pm 3$ & $79 \pm 3$ \\
Venous cross-sectional area $\left(\mathrm{cm}^{2}\right)$ & $0.19 \pm 0.03$ & $0.20 \pm 0.03$ \\
Venous blood velocity (cm/second) & $3.3 \pm 0.3$ & $3.6 \pm 0.3$ \\
Venous blood flow (ml/minute) & $39.2 \pm 7.3$ & $47.4 \pm 8.4$ \\
\hline
\end{tabular}

Values presented as mean \pm standard error. Static elbow flexion at $35 \%$ of maximal voluntary contraction with $(E X+V I B)$ and without $(E X)$ vibration of the biceps brachii tendon.

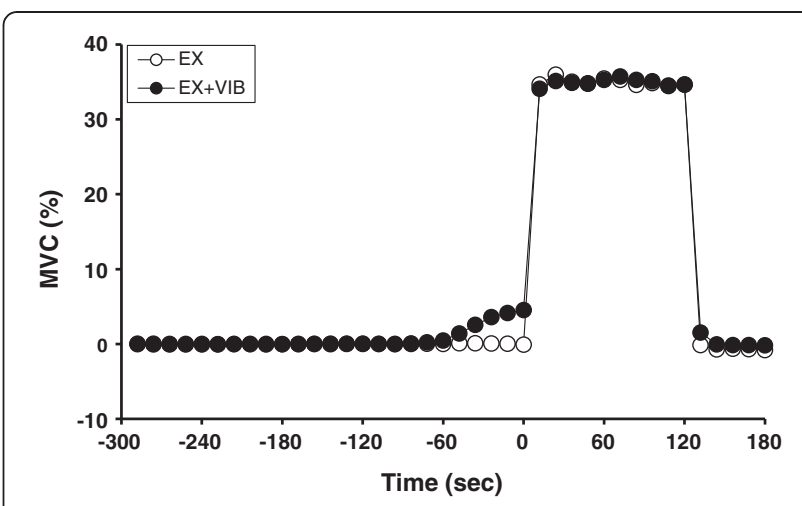

Figure 1 Time courses of muscle tension under each condition. Static elbow flexion at $35 \%$ of maximal voluntary contraction with $(E X+V I B)$ and without $(E X)$ vibration of the biceps brachii tendon. Data expressed as mean \pm standard error.

\section{Discussion}

The primary findings in this study were that $\mathrm{CSA}_{\text {vein }}$ decreased from baseline during static elbow flexion alone, although $\mathrm{CSA}_{\text {vein }}$ during static elbow flexion with tendon vibration did not change, and that $\mathrm{BF}_{\text {vein }}$ did not change significantly during static exercise with or without tendon vibration. These results suggest that a reduction in central command during static exercise with tendon vibration may attenuate the superficial venous vessel response of the resting limb during sustained static arm exercise.

Superficial venous vessel response may be controlled by both the sympathetic nervous system $[1,4,6,29]$ and changes in venous pressure related to alterations in blood flow and blood volume [30,31]. In our study, $\mathrm{BF}_{\text {vein }}$ did not change throughout the protocol during EX and EX + VIB (Figure 2E). In addition, $\Delta$ deoxy-Hb in the resting forearm did not change from baseline with static elbow flexion during both EX and EX + VIB (Figure 3D). Because the change in oxy-Hb and deoxy$\mathrm{Hb}$ is used to evaluate blood volume in arterial and venous vascular beds, respectively [32,33], it is speculated that the venous blood volume in the resting forearm was unchanged during static elbow flexion. In our study, therefore, the decrease in $\mathrm{CSA}_{\text {vein }}$ with exercise during EX may have been caused by sympathetic nervous system control. On the other hand, the difference in CSAvein between EX and EX + VIB during the recovery period might have been influenced by the change in venous blood volume but not by the sympathetic nervous system, because $\Delta$ deoxy- $\mathrm{Hb}$ of the resting forearm was also different between the two conditions (Figure 3D).

The concept of central command has been classically defined as a feed-forward control. Feed-forward characterization may be largely based on the immediate cardiovascular response to onset (or even anticipation) of exercise. In addition to feed-forward control, there is 


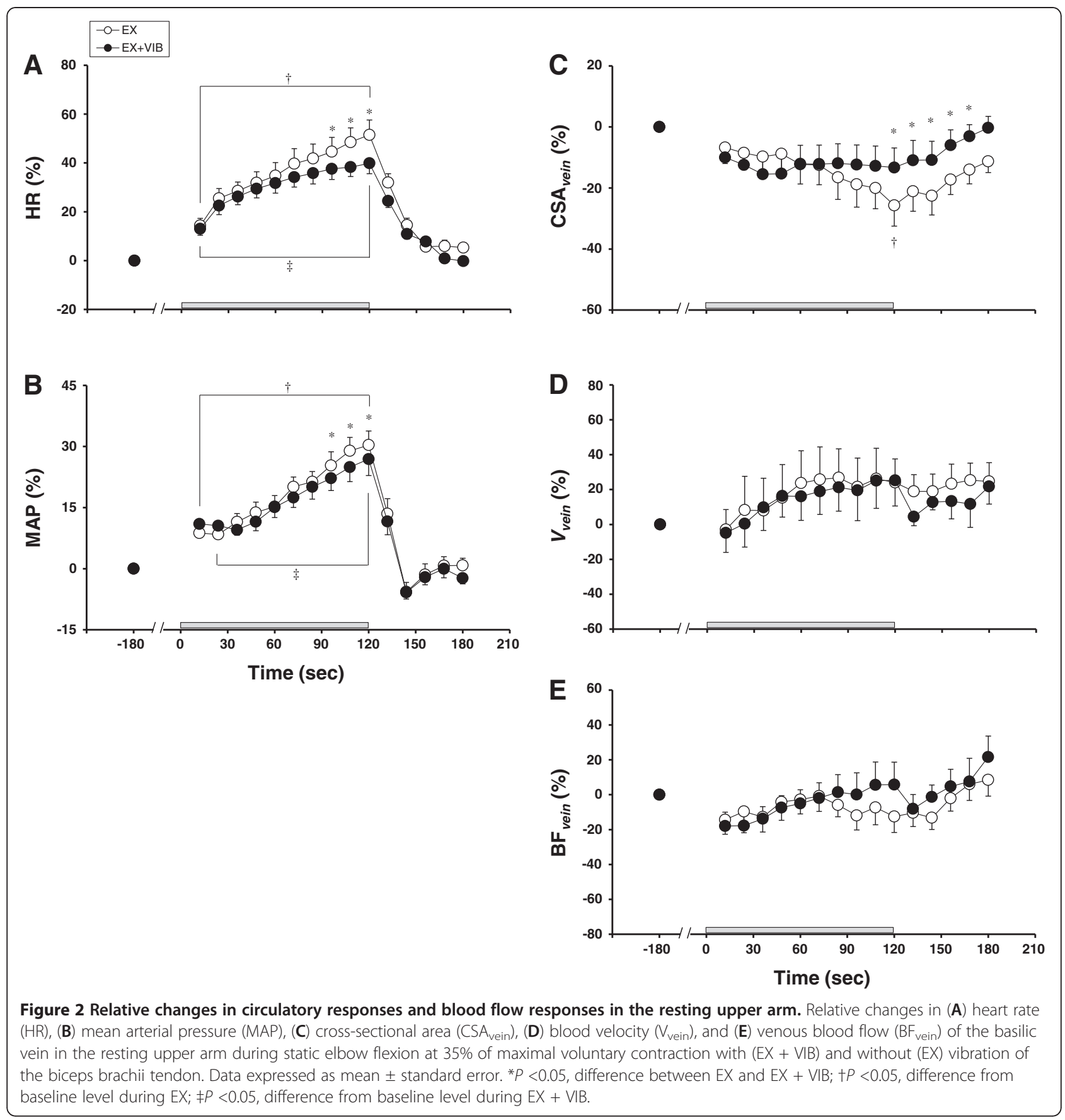

evidence that the effects of central command on cardiovascular responses are closely related to the intensity or perceived effort of the exercise [34,35]. Central command is there also proposed to be capable of functioning as feedback control, in which somatosensory signals arising from the working muscles may provide a feedback signal capable of influencing central command via alterations of perception of effort or effort sense $[19,20]$. The experimental model in our study might reflect central command that is defined as feedback control rather than feed-forward control, because the changes in HR and MAP, which are indexes of the cardiovascular response, were significantly lower during 96 to $120 \mathrm{sec}-$ onds of exercise in EX + VIB than in EX (Figure 2A,B). These results are in agreement with those of previous studies $[14,25,26]$. In addition, the magnitude of the central command response has been assessed using an individual's perception of effort sense during exercise, independent of force production [15,34]. Although the relationship between central command and RPE has not 


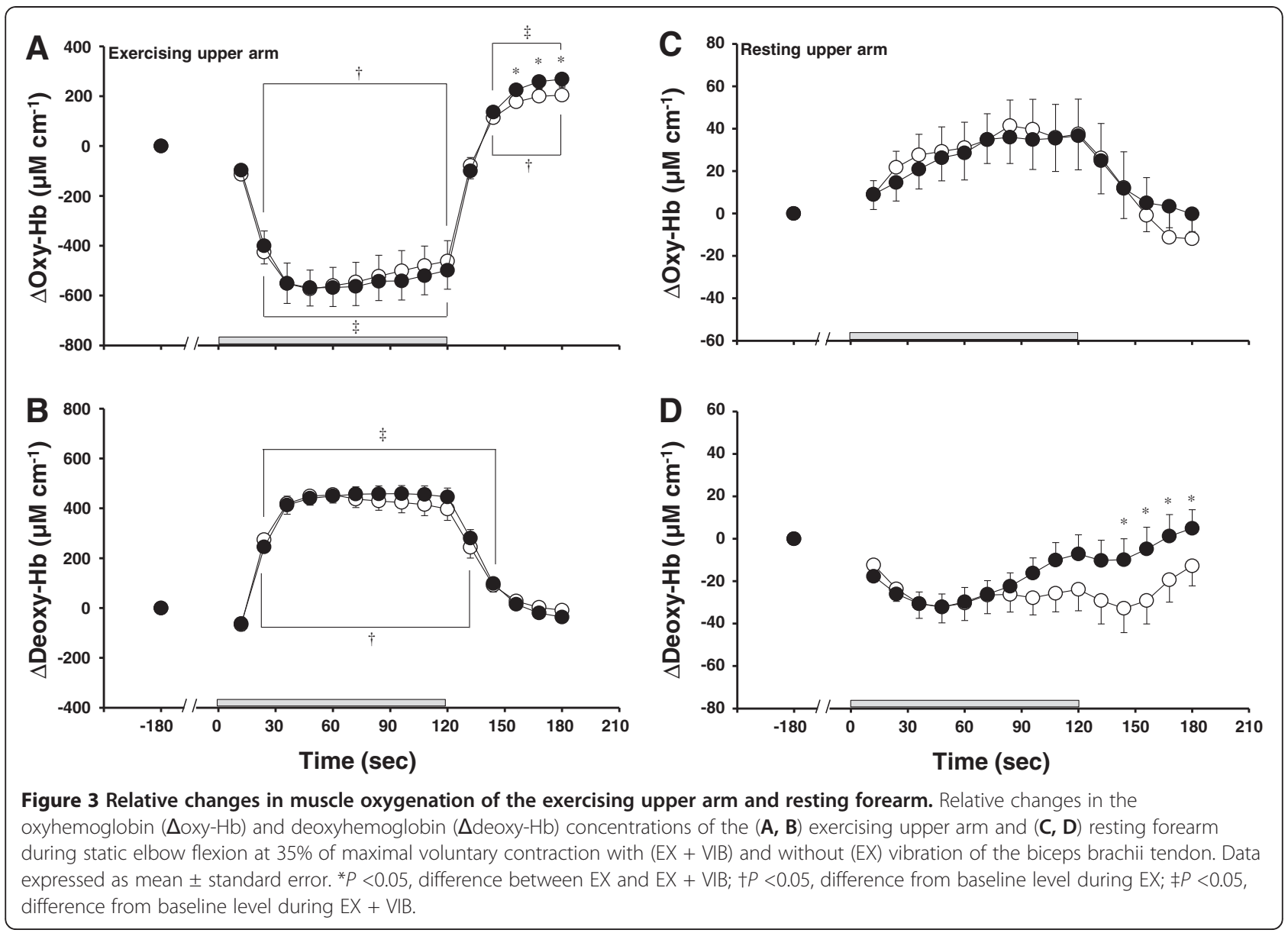

been clearly defined, the RPE scale [27] has been widely used to assess the level of central command. In the present study, RPE immediately after exercise was lower in EX + VIB than in EX, indicating that central command, which is defined as feedback control, might be lower in EX + VIB than in EX. Thus, related to the central command response that is defined as feedback control, $\mathrm{CSA}_{\text {vein }}$ was also smaller in EX than in EX + VIB during the latter half of the exercise. In addition, activation of the central command at the onset of static elbow flexion exercise in the present study, which indicated the feed-forward control, may have been too small to cause venoconstriction. If activation of central command at the onset of static elbow flexion exercise was enough to cause venoconstriction, the decrease in $\mathrm{CSA}_{\text {vein }}$ had to be obtained at the onset of exercise in both EX and EX + VIB.

Vibration is a powerful stimulus for primary muscle spindle afferents when applied to the biceps tendon during static exercise. When the biceps brachii was contracting, activation of its muscle spindle primary afferents provided reflex activation, which in turn aided voluntary tension development compared with contraction only of the biceps brachii. The afferent input of decreased voluntary tension during exercise with tendon vibration might thus cause interactions between perception of effort and central command, such that the activation of central command might alter [20].

The increase in sympathetic nervous system activity during exercise is caused not only by central command but also by the reflex neural mechanism that is activated by exercise (muscle mechanoreflex and muscle metaboreflex) $[9-11,13,16]$. Muscle-exerted tension during static elbow flexion did not differ between EX and EX + VIB (Figure 1), showing that the degree of activation of muscle mechanoreflex may be similar under both conditions. In addition, $\Delta$ deoxy-Hb concentration of the exercising upper arm was similar between EX and EX + VIB (Figure $3 \mathrm{~B}$ ). Because deoxy-Hb of exercising muscle is the index for oxygen consumption [36,37], the level of an exercise-induced metabolite accumulation during EX was expected to be equal to that found during EX + VIB, suggesting that the degree of activation of the muscle metaboreflex might not differ between EX and EX + VIB. In the present study, therefore, it is likely that the difference in $\mathrm{CSA}_{\text {vein }}$ during static exercise between 
EX and EX + VIB might not be due to the differences in activation of the reflex neural mechanism under different conditions.

Although the specific regions of the brain involved in exercise-related responses remain speculative, the following theory can be considered. Animal studies suggest that subthalamic regions are capable of generating both motor and cardiovascular responses [38]. In human studies, possible sites and neurocircuitry involving the insular cortex, sensorimotor cortex, anterior cingulate gyrus, medial prefrontal region and thalamic regions $[18,39-43]$, and the periaqueductal gray [44,45], have been suggested. In addition, a recent hypothesis concerning the neural circuit responsible for generating central command is as follows: cerebral cortical output is not an essential component for the generation of central command but does seem to require a process that triggers activity in neural circuit(s) in the caudal brain to generate central command, and the region from the caudal diencephalon to the rostral mesencephalon plays an important role in the generation of central command [46], because in the decerebrate animal study the renal sympathetic nerve activity and HR abruptly increased in association with the start of locomotion [47], and spontaneous motor activity and the associated cardiovascular response were lost after decerebration at the midcollicular level [48].

Stewart and colleagues reported that venoconstriction during static exercise, which occurs not only in the splanchnic area but also in the resting extremities, may contribute to an increase in venous return to the heart to increase cardiac output [49]. Taking into account previous studies, including our own, venoconstriction via central command might play a significant role in hemodynamics during exercise. However, because the relationship between venous return and venoconstriction is not obvious, further investigation is required.

\section{Limitations}

Several limitations should be considered when interpreting our results. First, due to the large compliance of veins, volume (that is, $\mathrm{CSA}_{\text {vein }}$ ) is dependent on the venous pressure level - but we did not measure venous pressure. As mentioned above, however, $\mathrm{BF}_{\mathrm{vein}}$ and $\Delta$ deoxy-Hb (an index of venous blood volume) of the resting forearm did not change from baseline during both EX and EX + VIB (Figures 2E and 3D). We therefore believe that the effect of venous pressure-dependent control was scarcely observed during exercise in this study. Second, we did not account for the menstrual cycle in female subjects. However, because EX and EX + VIB were carried out in same day, this effect may be negligible in our study.

\section{Conclusions}

Static elbow flexion with vibration of the biceps brachii tendon, which caused a decrease in central command during exercise, inhibited the increase in circulatory response and the decrease in $\mathrm{CSA}_{\text {vein }}$ in the resting upper arm when compared with static exercise alone, although $\mathrm{BF}_{\text {vein }}$ was similar during exercise both with and without tendon vibration. These findings suggest that central command may contribute to the superficial venous vessel response of the resting limb during sustained static elbow flexion.

\section{Abbreviations \\ $\mathrm{BF}_{\text {vein }}$ : Blood flow of the basilic vein; CSA: Cross-sectional area of basilic vein before correction; CSA $_{\text {vein: }}$ Accurate cross-sectional area of the basilic vein after correction; $\triangle$ : Change; EX: Elbow flexion without vibration; $E X+$ VIB: Elbow flexion with vibration; oxy-Hb: Oxyhemoglobin; deoxy- $\mathrm{Hb}$ : Deoxyhemoglobin; HR: Heart rate; MAP: Mean arterial pressure; MVC: Maximal voluntary contraction; RPE: Rating of perceived exertion; $V_{\text {vein: }}$ Blood velocity of the basilic vein.}

\section{Competing interests}

The authors declare that they have no competing interests.

\section{Authors' contributions}

AO designed and coordinated the study, carried out the experiment, and drafted the manuscript. KS participated in the design of the study and helped draft the manuscript. AH helped carry out the experiment. TS participated in the design of the study and helped draft the manuscript. All authors read and approved the final manuscript.

\section{Acknowledgements}

This study was supported by Grant-in-Aid for Scientific Research from the Japanese Ministry of Education, Science, Sports, and Culture (grant 22700593 to $\mathrm{AO}$ ) and a research grant from The Meiji-Yasuda Life Foundation of Health and Welfare.

\section{Author details}

${ }^{1}$ Research Institute of Physical Fitness, Japan Women's College of Physical Education, 8-19-1 Kitakarasuyama, Setagaya-ku, Tokyo 157-8565, Japan. ${ }^{2}$ Graduate school of Engineering, Toyo University, 2100 Kujirai, Kawagoe City, Saitama 350-8585, Japan

Received: 30 May 2012 Accepted: 19 October 2012

Published: 7 November 2012

References

1. Bevegård BS, Shepherd JT: Reaction in man of resistance and capacity vessels in forearm and hand to leg exercise. J Appl Physiol 1966, 21:123-132

2. Lorentsen E: The venomotor response to static muscular exercise. Scand Clin Lab Invest 1975, 35:789-794.

3. Seaman RG, Wiley RL, Zechman FW, Goldey JA: Venous reactivity during static exercise (handgrip) in man. J Appl Physiol 1973, 35:858-860.

4. Bevegård BS, Shepherd JT: Changes in tone of limb veins during supine exercise. J Appl Physiol 1965, 20:1-8.

5. Rowell LB, Brengelmann GL, Detry JM, Wyss C: Venomotor responses to rapid changes in skin temperature in exercising man. J Appl Physiol 1971, 30:64-71.

6. Zelis R, Mason DT: Comparison of the reflex reactivity of skin and muscle veins in the human forearm. J Clin Invest 1969, 48:1870-1877.

7. Abdel-Sayed WA, Abboud FM, Ballard DR: Contribution of venous resistance to total vascular resistance in skeletal muscle. Am J Physiol 1970, 218:1291-1295.

8. Vanhoutte PM, Lorenz RR: Effect of temperature on reactivity of saphenous, mesenteric, and femoral veins of the dog. Am J Physiol 1970, 218:1746-1750 
9. Mark AL, Victor RG, Nerhed C, Wallin BG: Microneurographic studies of the mechanisms of sympathetic nerve responses to static exercise in humans. Circ Res 1985, 57:461-469.

10. Rowell LB, O'Leary DS: Reflex control of the circulation during exercise: chemoreflex and mechanoreflexes. J Appl Physiol 1990, 69:407-418.

11. Victor RG, Bertocci LA, Pryor SL, Nunnally RL: Sympathetic nerve discharge is coupled to muscle cell $\mathrm{pH}$ during exercise in humans. J Clin Invest 1988, 82:1301-1305.

12. Victor RG, Pryor SL, Secher NH, Mitchell JH: Effects of partial neuromuscular blockade on sympathetic nerve responses to static exercise in humans. Circ Res 1989, 65:468-476.

13. Wallin BG, Victor RG, Mark AL: Sympathetic outflow to resting muscles during static handgrip and postcontraction muscle ischemia. Am J Physiol 1989, 256:H105-H110.

14. Goodwin GM, McCloskey DI, Mitchell JH: Cardiovascular and respiratory responses to changes in central command during isometric exercise at constant muscle tension. J Physiol 1972, 226:173-190.

15. Mitchell JH, Reeves DR Jr, Rogers HB, Secher NH, Victor RG: Autonomic blockade and cardiovascular responses to static exercise in partially curarized man. J Physiol 1989, 413:433-445.

16. Mitchell $\mathrm{JH}$ : Neural control of the circulation during exercise. Med Sci Sports Exerc 1990, 22:141-154.

17. Duprez DA, De Buyzere M, De Sutter JM, Deman SA, De Pue NY, Clement $\mathrm{DL}$ : Venous responses to rhythmic exercise in contralateral forearm and calf. Eur J Appl Physiol Occup Physiol 1992, 65:94-98.

18. Williamson JW, Fadel PJ, Mitchell JH: New insights into central cardiovascular control during exercise in humans: a central command update. Exp Physiol 2006, 91:51-58.

19. Amann M, Proctor LT, Sebranek JJ, Eldridge MW, Pegelow DF, Dempsey JA: Somatosensory feedback from the limbs exerts inhibitory influences on central neural drive during whole body endurance exercise. J Appl Physiol 2008, 105:1714-1724.

20. Williamson JW: The relevance of central command for the neural cardiovascular control of exercise. Exp Physiol 2010, 95:1043-1048.

21. de Groot PC, Bleeker MW, Hopman MT: Ultrasound: a reproducible method to measure conduit vein compliance. J Appl Physiol 2005, 98:1878-1883

22. Ooue A, Ichinose-Kuwahara T, Shamsuddin AK, Inoue Y, Nishiyasu T, Koga S, Kondo $\mathrm{N}$ : Changes in blood flow in a conduit artery and superficial vein of the upper arm during passive heating in humans. Eur J Appl Physiol 2007, 101:97-103.

23. Ooue A, Ichinose TK, Inoue $Y$, Nishiyasu T, Koga S, Kondo N: Changes in blood flow in conduit artery and veins of the upper arm during leg exercise in humans. Eur J Appl Physiol 2008, 103:367-373.

24. Young CN, Prasad RY, Fullenkamp AM, Stillbower ME, Farquhar WB, Edwards DG: Ultrasound assessment of popliteal vein compliance during a short deflation protocol. J Appl Physiol 2008, 104:1374-1380.

25. Sato K, Sadamoto T, Ueda-Sasahara T, Shibuya K, Shimizu-Okuyama S, Osada T, Kamo M, Saito M, Kagaya A: Central command and the increase in middle cerebral artery blood flow velocity during static arm exercise in women. Exp Physiol 2010, 94:1132-1138.

26. Ogoh S, Wasmund WL, Keller DM, O-Yurvati A, Gallagher KM, Mitchell JH, Raven PB: Role of central command in carotid baroreflex resetting in humans during static exercise. J Physiol 2002, 543:349-364.

27. Borg GA: Perceived exertion: a note on 'history' and methods. Med Sci Sports 1971, 5:90-93.

28. Saito M, Mano T, Iwase S: Sympathetic nerve activity related to local fatigue sensation during static contraction. J Appl Physiol 1989, 67:980-984.

29. Duggan JJ, Love $\mathrm{VL}$, Lyons $\mathrm{RH}$ : A study of reflex venomotor reactions in man. Circulation 1953, 7:869-873.

30. Noble BJ, Drinkhill MJ, Myers DS, Hainsworth R: Reflex control of splanchnic blood volume in anaesthetized dogs. J Physiol 1998, 513:263-272.

31. Öberg B: The relationship between active constriction and passive recoil of the veins at various distending pressures. Acta Physio/ Scand 1967, 71:233-247.

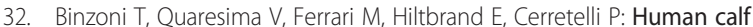
microvascular compliance measured by near-infrared spectroscopy. J Appl Physiol 2000, 88:369-372.
33. Hachiya T, Blaber AP, Saito M: Changes in superficial blood distribution in thigh muscle during LBNP assessed by NIRS. Aviat Space Environ Med 2004, 75:118-122.

34. Leonard B, Mitchell JH, Mizuno M, Rube N, Saltin B, Secher NH: Partial neuromuscular blockade and cardiovascular responses to static exercise in man. J Physiol 1985, 359:365-379.

35. Gandevia SC, Killian K, McKenzie DK, Crawford M, Allen GM, Gorman RB, Hales JP: Respiratory sensations, cardiovascular control, kinaesthesia and transcranial stimulation during paralysis in humans. J Physiol 1993, 470:85-107.

36. Koga S, Poole DC, Ferreira LF, Whipp BJ, Kondo N, Saitoh T, Ohmae E, Barstow TJ: Spatial heterogeneity of quadriceps muscle deoxygenation kinetics during cycle exercise. J Appl Physiol 2007, 103:2049-2056.

37. Saitoh T, Ferreira LF, Barstow TJ, Poole DC, Ooue A, Kondo N, Koga S: Effects of prior heavy exercise on heterogeneity of muscle deoxygenation kinetics during subsequent heavy exercise. Am J Physiol Regul Integr Comp Physiol 2009, 297:R615-R621.

38. Eldridge FL, Millhorn DE, Waldrop TG: Exercise hyperpnea and locomotion: parallel activation from the hypothalamus. Science 1985, 211:844-846.

39. King AB, Menon RS, Hachinski V, Cechetto DF: Human forebrain activation by visceral stimuli. J Comp Neurol 1999, 413:572-582.

40. Nowak M, Holm S, Biering-Sørensen F, Secher NH, Friberg L: 'Central command' and insular activation during attempted foot lifting in paraplegic humans. Hum Brain Mapp 2005, 25:259-265.

41. Thornton JM, Guz A, Murphy K, Griffith AR, Pedersen DL, Kardos A, Leff A Adams L, Casadei B, Paterson DJ: Identification of higher brain centres that may encode the cardiorespiratory response to exercise in humans. J Physiol 2001, 533:823-836.

42. Williamson JW, McColl R, Mathews D, Mitchell JH, Raven PB, Morgan WP: Hypnotic manipulation of effort sense during dynamic exercise: cardiovascular responses and brain activation. J Appl Physiol 2001, 90:1392-1399.

43. Williamson JW, McColl R, Mathews D, Mitchell JH, Raven PB, Morgan WP: Brain activation by central command during actual and imagined handgrip under hypnosis. J Appl Physiol 2002, 92:1317-1324.

44. Green AL, Wang S, Purvis S, Owen SL, Bain PG, Stein JF, Guz A, Aziz TZ, Paterson DJ: Identifying cardiorespiratory neurocircuitry involved in central command during exercise in humans. J Physiol 2007, 578:605-612.

45. Green AL, Paterson DJ: Identification of neurocircuitry controlling cardiovascular function in humans using functional neurosurgery: implications for exercise control. Exp Physiol 2008, 93:1022-1028.

46. Matsukawa K: Central command: control of cardiac sympathetic and vagal efferent nerve activity and the arterial baroreflex during spontaneous motor behaviour in animals. Exp Physiol 2012, 97:20-28.

47. Matsukawa K, Murata J, Wada T: Augmented renal sympathetic nerve activity by central command during overground locomotion in decerebrate cats. Am J Physiol 1998, 275:H1115-H1121.

48. Hayashi N: Exercise pressor reflex in decerebrate and anesthetized rats. Am J Physiol Heart Circ Physiol 2003, 284:H2026-H2033.

49. Stewart JM, Montgomery LD, Glover JL, Medow MS: Changes in regional blood volume and blood flow during static handgrip. Am J Physiol Heart Circ Physiol 2007, 292:H215-H223.

\section{doi:10.1186/1880-6805-31-29}

Cite this article as: Ooue et al:: Tendon vibration attenuates superficial venous vessel response of the resting limb during static arm exercise. Journal of Physiological Anthropology 2012 31:29. 